Meta

Journal des traducteurs

Translators' Journal

\title{
Pour traduire " Un coup de dés... »
}

\section{François Peraldi}

Volume 23, numéro 1, mars 1978

La traduction poétique

URI : https://id.erudit.org/iderudit/003814ar

DOI : https://doi.org/10.7202/003814ar

Aller au sommaire du numéro

\section{Éditeur(s)}

Les Presses de l'Université de Montréal

\section{ISSN}

0026-0452 (imprimé)

1492-1421 (numérique)

Découvrir la revue

Citer cet article

Peraldi, F. (1978). Pour traduire « Un coup de dés... ». Meta, 23(1), 109-123.

https://doi.org/10.7202/003814ar d'utilisation que vous pouvez consulter en ligne.

https://apropos.erudit.org/fr/usagers/politique-dutilisation/ 


\section{Pour traduire "Un coup de dés..."}

A Roland Barthes en signe d'amitié.

\section{Préambule}

Dans ce qui suit nous avons imaginé qu'un traducteur serait assez fou pour entreprendre de traduire $U n$ coup de dés de Stéphane Mallarmé. Et, pour compliquer les choses, nous avons fait précéder notre amorce de traduction du Coup de dés de ce que nous appelons une "lectécriture» de Mallarmé. Lectécriture que nous devons à Julia Kristeva ${ }^{1}$. Il nous semble en effet que le traducteur ne saurait faire l'économie de la plus solide des cultures, de la plus actuelle, que ce soit dans le champ de la linguistique ou, au-delà, de la sémiotique de la sémanalyse et de la critique littéraire quelle qu'en soit la forme. Pourquoi de la plus actuelle? parce que nous pensons que les cuvres vivent. Leur vie est ce qu'en linguistique on nomme la "valeur ${ }^{2}$ ", c'est-à-dire ce sens toujours en devenir, cette signification devrions-nous dire, qui vient au texte du contexte dans lequel il est pris, de $\mathrm{l}^{\prime}$ " intertexte ${ }^{3}$ 》. Il apparaitra vite que le contexte dans lequel je lis aujourd'hui Mallarmé n'a plus rien à voir avec celui dans lequel Henri Mondor ou Paul Valéry ont pu le lire et que, de ce fait, je ne le lis plus comme eux ont pu le lire et nous en proposer le témoignage. Le texte mallarméen pris dans le contexte des textes de Philippe Sollers, Jacques Derrida, Julia Kristeva, voire même dans l'horizon de ceux d'Antonin Artaud ou de Louis Wolfson ${ }^{4}$ n'a plus du tout la même valeur que celle qu'il recevait du contexte des symbolistes de son temps.

Moi, traducteur, je ne traduis pas Mallarmé pour un lecteur comme pourrait l'avoir été Henti de Régnier, mais pour le lecteur d'aujourd'hui, un lecteur qui lirait comme Roland Barthes peut-être lectécrit pour nous ce qu'il aime. Pensez au Plaisir du texte ${ }^{5}$ ! Car ce serait un peu le sens que nous donnons à ce terme de lectécriture : une écriture qui nous dirait le plaisir du texte, le

1. Julia Kristeva, la Révolution du langage poétique, Seuil, Paris, 1974.

2. Sur cette question absolument essentielle pour Ferdinand de Saussure, cf. Cours de linguistique générale, Payot, Paris, 1973 , p. 158 et ss., \& la valeur linguistique considérée dans son aspect conceptuel \$.

3. Nous devons la notion d'intertexte à Julia Kristeva et l'on sait assez la fortune de ce mot nouveau dans les élans structuralistes et néo-structuralistes français. Cf. Semiotiké, "recherches pour une sémanalyse ", Seuil, 1969, p. 113. Le texte clos.

4. Pourquoi Artaud et Wolfson? précisément parce qu'ils ont servi de "modèles *, à leur corps défendant si je puis dire, pour les poètes qui ont fait exploser les conceptions classiques de la langue et de l'écriture poétique.

5. Roland Barthes, le Plaisir du texte, Seuil, Paris, 1973. 
plaisir de lire pour écrire, d'écrire une lecture. Ma traduction n'a pas à tenir compte avant tout de ce soi-disant «sens»du texte, dont tout linguiste sait bien qu'il n'existe pas de façon intangible mais bien plutôt de façon contingente, c'est-à-dire secondaire au regard de la valeur qui elle-même est une fonction par excellence variable du contexte dans lequel je traduis mon texte, dans lequel je le lis, un contexte qui n'est qu'une variable de l'histoire elle-même.

\section{D'une lectécriture de Mallarmé...}

Nous avons introduit dans notre thèse de doctorat, d'où ce petit essai est extrait, une notion qui flottait dans l'air depuis quelque temps, celle de lectécriture. Si nous tentons de la reprendre ici, c'est parce qu'il nous semble qu'elle peut présenter un certain intérêt pour le traducteur littéraire dont le statut et la responsabilité au regard des textes qu'il traduit sont d'une toute autre nature que ceux du technicien en traduction qui lentement se fane dans les bureaux.

On demande au traducteur littéraire de savoir faire au moins trois choses : lire, écrire et, entre les deux, traduire. Mais pour lui, traduire doit prendre tous les sens que cette fonction doit à l'étymologie du terme qui la désigne.

Que signifie traduire?

Le dictionnaire étymologique de la langue française de Bloch et Von Wartburg nous dit : «Traduire : faire passer d'une langue dans une autre 1534. Traducteur, 1540 (E. Dolet); traduction: emprunté des mots latins, traducere (ou de l'italien tradurre?), proprement «faire passer», traductor, traductio; les deux derniers n'ont pas le sens du français; ils ont été pris comme substantifs de traducere pour servir de noms d'agent et d'action à traduire. »

En effet les sens les plus usités de traductio renvoient au trajet d'un criminel vers le lieu de son supplice (Sénèque), sens que nous retrouvons dans l'expression : "traduire en justice», ou bien encore le transport d'un peuple tout entier vers une autre province, comme ce fut le cas pour certains colons français au Canada, il y a de cela quelques siècles. C'est aussi l'action de faire passer d'un ordre à un autre, ou encore, l'adoption plébéienne d'un misérable.

Nous trouvons également chez Cicéron un sens qui nous retiendra : métonymie, et aussi le cours de la marche du temps.

Continuons à lire Bloch et Von Wartburg : «Celui-ci (le mot traduire) remplace l'ancien français translater conservé dans l'anglais to translate.»

Ainsi la traduction est-elle aussi translation. Selon Bloch et Von Wartburg : « la translation en 1330 était un terme juridique signifiant l'action de faire passer des personnes dans une autre situation. Au XvIr ${ }^{8}$ siècle ce mot signifie encore «transport d'un corps». Il a signifié aussi au xIr siècle et jusqu'au $\mathrm{XVI}^{\mathrm{e}}$ siècle : «traduction ».

«Il dérive du latin translatio «transport, transfert» dérivé de translatus participe passé de transferre. »

À l'origine du mot traduction nous trouvons donc bien une double racine, l'une dont le mot traduire est directement dérivé : traducere mais aussi tra- 
ductio; l'autre dont le mot traduction dérive indirectement par assimilation sémantique avec translation : transferre, translatio.

Traducere signifie :

- conduire au-delà, d'un lieu à un autre.

- au figuré : faire passer (d'un ordre à un autre), élever à une dignité.

- donner un spectacle, exposer à la risée, montrer, publier, divulguer.

- passer le temps,

- et ceci qui nous intéresse encore davantage : faire passer un mot c'un sens à un autre ou d'une langue à une autre (Aulu-Gelle). Nous avons déjà souligné le sens de «métonymie» que Cicéron donne à traäuctio.

Transferre signifie :

- (Suétone) : transcrire.

- (Cicéron), (Quintilien) : faire passer d'une langue dans une autre, traduire.

- (Cicéron) : différer, ajourner, remettre.

\section{Translatio signifie :}

- transplanter, greffer par incision.

- (Suétone) : changement de domicile.

- (Quintilien) : traduction.

- (Cicéron), (Quintilien) : métaphore.

- également, copie et métathèse.

Par sa double racine qui le lie à traductio et à translatio, la traduction se trouve donc dans le rapport le plus étroit avec ce que Roman Jakobson désigne comme les deux grands axes de fonctionnement du langage : la métaphore et la métonymie.

Par le biais de l'étymologie, la traduction rejoint bien (et même nous pouvons dire se conjoint avec la propriété transuasive ${ }^{6}$ du signe d'être remplaçable par un signe équivalent (métaphore) ou plus développé (métonymie), telle qu'elle en constitue pour Charles Sanders Peirce, la définition.

L'opération traduisante travaille le signe aussi bien sur l'axe de la métaphore que sur celui de la métonymie : elle est le travail du signe. Le mot travail étant ici à prendre dans tous les sens du terme et plus particulièrement dans celui d'engendrement que l'on retrouve dans le travail de la femme qui accouche.

Il n'est pas inutile de souligner que nous retrouvons dans les variantes du sens des deux racines les différentes connotations dont la traduction, au cours des siècles s'est trouvée marquée.

6. On trouvera tous les développements concernant la théorie du signe chez Peirce dans ses "Collected Papers », t. 1, vol. 2, Harvard University Press (Cambridge), 1963. La meilleure étude qui en ait été faite reste, à date, celle de D. Greenlee, Peirce's Concept of Sign, Mouton, La Haye, 1973. 
Ainsi la traduction comme déshonorant le texte original, qu'il donne en spectacle, qu'il divulgue, reproche adressé aux premiers traducteurs de la Bible (troisième sens de traducere).

La traduction comme méfait, comme crime : "traduttore, traditore » ainsi lié au sens de traductio : le trajet d'un criminel, d'un traître, vers le lieu de son châtiment.

Mais il y a tout de même un autre des sens originels qui nous intéresse plus particulièrement, celui que Cicéron donne à transferre : ajourner, différer. Translater, traduire, c'est aussi différer. La traduction se joue peut-être tout entière entre les bords de la différence (transporter d'un ordre à un autre, d'un bord à l'autre), mais elle est aussi différance, ce qui nous rapproche étrangement de la définition déridienne de l'écriture, si proche de ce que l'on peut entendre par «traduction »c'est-à-dire l'activité qui, par excellence et constamment, diffère.

Enfin, si la traduction oscille entre la métaphore et la métonymie, si l'opération traduisante est de ce fait une sorte d'oscillation métaphoro-métonymique, cette dernière définition la place de façon tout à fait précise dans le registre du désir et de la jouissance tels qu'ils sont définis, après Freud, dans la théorie lacanienne ${ }^{7}$. Si le Plaisir du texte est lié, comme nous le propose Roland Barthes, à ce que je nomme sa lectécriture, sa traduction en est la jouissance, c'est-à-dire également la destruction.

Posons comme thèse, au sens althusserien de ce terme ${ }^{8}$, que : la traduction ne saurait s'originer que de la jouissance qui, au-delà du plaisir du texte (la lectécriture), la fonde.

Lectécrire, c'est être à même d'écrire la lecture que l'on a pu faire d'un texte afin d'élaborer dans cette écriture même le plaisir du texte. Ce faisant, le critique - et je pense à Roland Barthes - tout comme le traducteur - et je pense à Marguerite Yourcenar — sont des lecteurs privilégiés en ce sens que par eux et pour eux, la lecture est toujours une relecture, condition minimale pour quiconque veut saisir la structure du texte dans lequel il s'aventure, c'est-à-dire aussi bien celle de son propre principe de plaisir. Or, la relecture - même d'un même texte - ne peut pas ne pas nous introduire à la notion de valeur. La valeur du texte que nous relisons n'est en effet plus la même que celle de ce même texte lorsque nous l'avons $\mathrm{lu}$, puisque lorsque nous le relisons, sa valeur s'augmente grâce au nouveau contexte que constitue le souvenir que nous avons de sa première lecture. La lecture de $S / Z$ de Roland Barthes ${ }^{9}$ serait le meilleur exemple de ce que je souligne ici. En d'autres termes, en relisant le texte, nous le ré-écrivons en quelque sorte. La relecture - cette pratique privilégiée du critique et du traducteur - est une ré-écriture.

7. Il est possible d'aborder l'étude des rapports du langage et du désir chez Lacan en lisant ce texte aujourd'hui facile mais qui en son temps fut si scandaleux qu'il en secoua le monde psychanalytique jusqu'en ses fondements : "Fonction et champ du langage et de la parole en psychanalyse ", in : Ecrits, Seuil, Paris, 1966.

8. Louis Althusser, in : Positions, Editions sociales, Paris, 1976, l'article intitulé « Appa-

reils d'Etat et appareils idéologiques d'Etat ».

9. Roland Barthes, $S / Z$, Seuil, Paris, 1970. 
C'est, croyons-nous, d'une longue cohabitation avec le texte mallarméen que s'origine la lectécriture que nous en propose Julia Kristeva dans la Révolution du langage poétique et si nous voulons en reprendre ici les étapes c'est parce qu'elle éclaire bien cette valeur du texte mallarméen dans la langue dite d'arrivée, dans le même temps qu'elle en dégage la structure spécifique.

Pour le lecteur non-prévenu, la première lecture, le premier regard jeté sur la «partition» du Coup de dés est comme un choc et vient comme « une grande surprise »; "il me semble, écrit Paul Valéry ${ }^{10}$ qui fut le premier à le lire, voir la figure d'une pensée, pour la première fois placée dans notre espace... Cette fixation sans exemple me pétrifiait ». C'est que tout dans ce texte déroute, « je me sentais livré à la diversité de mes impressions, saisi par la nouveauté de l'aspect, tout divisé de doutes, tout remué de développements prochains $\gg$. La disposition graphique même de ce texte, en première lecture, brouille, rend les enchaînements incertains, imposant au regard ces constellations qui s'appréhendent en surface - à cette lecture superficielle dont parle quelque part Valéry - et, simultanément, dans leur pluralité.

Lectécrire Un coup de dés, c'est pour Julia Kristeva dégager les processus organisateurs de ce texte, «les processus syntaxiques, constitutifs de suites phrastiques ${ }^{11}{ }^{»}$.

Chez Mallarmé la linéarité syntaxique habituelle (celle qui constitue le support nécessaire de la monosémie et de la vérité tout autant que l'unique garantie de toute traduction possible du sens, c'est-à-dire la traduction au sens classique du terme) est sujette à deux groupes de modifications «propres au langage même de Mallarmé ${ }^{12}$ ».

Le premier groupe de modifications est constitué par « des inversions, des appositions et des ellipses dont on peut reconstituer la structure sous-jacente ${ }^{13} \gg$. Structure évidemment sujette à de très nombreuses transformations que d'autres processus viennent en quelque sorte accroître ( $\ll$ transposition, condensation, répétition 》). L'effet poétique serait alors la résultante de la conjonction de cette structure sous-jacente reconstituable à sa transformation finale par inversion, apposition, ellipse.

Ne prenons qu'un exemple : «... à n'ouvrir la main crispée par-delà l'inutile agonie ${ }^{14} \gg$ que l'on peut aisément reconstituer en abolissant les inversions : à ne pas ouvrir la main crispée par-delà l'agonie inutile. Dans ce cas l'effet de l'inversion est une certaine préciosité. Notons dès le début une élision du verbe (le plus souvent d'ailleurs il s'agit du verbe être) : «... jamais quand bien même lancé $=\ldots$ jamais quand bien même serait-il lancé. »

C'est presque toujours l'élision qui en fait tend à dominer dans ce processus par rapport à la structure sous-jacente reconstituable, l'élision et la conden-

10. Paul Valéry, Ecrits divers sur Stéphane Mallarmé, Gallimard, Paris, 1950.

11. Toutes les citations de Julia Kristeva qui suivent sont extraites de la Révolution du langage poétique. Dans les notes suivantes nous n’indiquerons donc que le nom de Kristeva et le numéro de la page.

12. Kristeva, p. 269.

13. Kristeva, p. 269.

14. Stéphane Mallarmé, Euvres completes, Gallimard, Pléiade, Paris, p. 464. 
sation de ces éléments-nœuds qui sont soit des verbes à l'infinitif ou également souvent au participe passé ou présent, soit des noms. L'élision d'éléments de liaison rendant toujours la structure de la phrase plus ambiguë, plus fragile et, de ce fait, la polysémie des éléments qui la constituent plus forte, plus agissante et ce d'autant plus que le texte est moins linéarisé.

Le deuxième groupe de modifications - qui n'est peut-être qu'une forme extrême du premier groupe - est fait « d'emboîtements infinis et de suppressions non-recouvrables dont on ne peut reconstituer la structure sous-jacente et ce, pour deux raisons :

- soit à cause de «l'élision des pro-formes à la base »,

- soit « parce que les branchements sont en nombre indéfini et plurilatéraux ${ }^{15}$ 》.

Ici la modification n'est plus de surface, mais elle atteint le texte à ce point en profondeur, la syntaxe n'étant plus là pour conserver une quelconque linéarité phrastique, que peuvent intervenir les phénomènes de déplacement, de transpositions qui ne sont plus ici combinés à une structure sous-jacente afin de provoquer l'effet poétique dont il était question dans le premier groupe de transformations, mais qui s'y substituent pour introduire ces opérations sémiotiques pré-syntaxiques qui font exploser le sujet et le parcellarisent.

On trouvera toutes sortes d'exemples de ces emboîtements et de ces suppressions qui ne se prêtent pas à la reconstitution d'une structure sous-jacente et appellent à l'intervention des processus pré-syntaxiques dans l'étude de Kristeva. Notons que ces processus sont étrangement semblables à ceux du travail du rêve et qu'il faut déconstruire au cours de leur analyse lorsqu'on fait retour sous le sens manifeste au texte latent qui l'anime, il s'agit de la transposition, la condensation et le déplacement.

Afin de repérer l'ensemble de ces modifications, il convient de se donner un point de départ, d'une "phrase matrice», "phrase capitale » dès le titre introduite et continuée : «un coup de dés jamais n'abolira le hasard ${ }^{16}{ }$. Dans une perspective peircéenne nous pourrions dire que le Signe : «jamais un coup de dés n'abolira le hasard» expose dans le poème sa propriété essentielle (et qui par conséquent en est également la définition) d'être remplaçable par un autre Signe qui, dans le cas qui nous occupe présentement, n'est pas équivalent, mais plus développé. Développement qui n'est par ailleurs pas linéaire mais spatial et pluri-dimensionnel et qui met en jeu tout ce qu'il en est du rapport du Signe à l'écriture, d'une part, et du procès de la signifiance dans son ensemble du sujet. Un développement qui dans un sens se fonde sur l'opération traduisante au sens le plus global du terme.

Le développement du Signe, la phrase capitale du texte de Mallarmé, pour Kristeva : la phrase matrice, se fait par enchâssement, peut-être l'enchâssement « des reflets prismatiques de l'Idée », pour reprendre ici le texte même de Mallarmé, ou bien encore en d'autres termes de toutes les traductions possibles de

15. Kristeva, p. 269.

16. Mallarmé, op. cit., p. 455 . 
l'ensemble des sèmes initiaux contenus dans la phrase capitale comme des germes et qui attendent d'être reformuler, qui attendent leur germination.

Le premier enchâssement : "quand bien même lancé dans des circonstances éternelles », se rattache à la matrice d'abord sémantiquement du fait qu'il contient le verbe «lancer» élidé du syntagme nominal de la matrice «Un coup de dés ». Ce syntagme nominal est lui-même le résultat d'une triple transformation à partir d'une phrase constituante : «X lance les dés» qui subit une première transformation passive : «les dés sont lancés par $X$ », puis une seconde transformation par élision du syntagme nominal indéfini «par $X$ », et enfin la troisième transformation de nominalisation : «sont lancés» devient : * un coup ».

En fait, cette première séquence ne se rattache pas seulement sémantiquement à la matrice, mais aussi à l'aide du « déterminant conditionnel quand bien même ». Toutefois, et c'est cette duplicité de l'enchâssement que nous voulons prendre en considération, celui-ci peut également se faire par rapport au verbe de la matrice «n'abolira» comme syntagme adverbial «n'abolira le hasard, quand bien même... »

Peut-être le traducteur qui nous a suivi jusqu'ici commence-t-il à entrevoir la nécessité, mais aussi la complexité d'une telle lecture pour lui qui a été dressé à traduire le sens.

Cette double série de modifications syntaxiques opère une véritable mise en question du Signe comme élément constitutif du texte. En effet, et c'est en tout cas ce que nous montrait Kristeva dans un article de $1972^{17}$, si au regard de la phrase comme unité maximale de la linguistique de la communication le Signe reste l'élément constitutif d'un système, au regard du texte ce n'est ni la phrase ni le signe qui en sont les éléments constituants et il convient d'envisager une autre unité, car la proposition dans le texte est sans cesse modifiée, cassée, disloquée, inreconstituable, même dans sa forme la plus simple.

Prenons un exemple : «L'Abîme blanchi étale furieux». Qu'est-ce que le terme «étale»? un adjectif? une forme verbale de la troisième personne du singulier? un substantif?

Quant à «furieux », se rapporte-t-il à «Abîme» ou à «étale »? Du fait de l'étoilement et du déchaînement du texte, il est impossible de clore ces questions par une quelconque réponse. Il apparaît donc que ce ne sont pas des propositions que l'on pourrait saisir même dans leur forme la plus simple, mais qu'il s'agit plutôt de ce que Julia Kristeva nomme des \& complexes signifiants », eux-mêmes constitués d'éléments minimaux qu'elle nomme dans une perspective toute freudienne : "différentielles signifiantes». Il s'agit de ces points de refonte, au sein du texte, du signifiant et du signifié. Les différentielles signifiantes pourraient être également ces mots dont Antonin Artaud ${ }^{1.8}$ utilisait la chair même pour écrire un livre comme Letura d'Eprahi

17. Julia Kristeva, Quelques problèmes de sémiotique littéraire *, in : Essais de sémiotique littéraire, Larousse, Paris, 1972, p. 208-234.

18. Antonin Artaud, Euvres complètes, t. IX, Lettres de.Rodez, Gallimard, Paris, 1975. 
Falli Tetar Fendi Photia o Fotre Indi, ce livre ancien et perdu dont il parle à André Parisot dans ses lettres de Rodez. Ce seraient aussi peut-être, plus proches de nous, quelques-unes de ces pages mystérieuses de Gauvreau. Ou bien encore ces traces brunâtres, précises comme des signes ou ces mots anglais sales comme des traces de merde qui constituent les admirables toiles de Heybour, mais aussi encore "le mot anglais ${ }^{19}$ 》 de Stéphane Mallarmé lui-même : «ce mot qui présente dans ses voyelles et ses diphtongues comme une chair, et dans ses consonnes comme une ossature délicate à disséquer $»$.

Définie par Julia Kristeva, la différentielle signifiante est doublement caractérisée :

1) elle est une refonte du signifiant et du signifié ;

2) elle contient :

a) tous les homonymes,

b) tous les synonymes,

c) toutes les différentes acceptions mythiques, religieuses ou sociales qu'elle est susceptible d'avoir dans les différentes langues.

J'y ajouterai quant à moi qu'elle est le représentant de par son excès ou son absence de la pulsion sémiotique, imposant au texte telle pulsionnalité, anale par exemple, de par son insistance :

\author{
Potam am cram \\ Katanam anankreta \\ Karaban Kreta \\ tanamam anangteta \\ konamam kreta.. \\ (Antonin Artaud)
}

$\mathrm{Au}$ niveau du texte, et si l'on veut faire un parallèle qui n'est absolument pas nécessaire, avec l'énoncé dont l'unité minimale est la phrase, l'unité minimale est le complexe signifiant qui ne se construit plus par l'assemblage d'un sujet et d'un prédicat, ni même à partir d'une structure de base sujet-prédicat sous-jacente et toujours reconstituable grâce aux opérations normativantes de la grammaire transformationnelle. Ce complexe signifiant est bien plutôt composé « d'un modifiant et d'un modifié » dont Kristeva nous propose la grille d'analyse dans ses recherches pour une sémanalyse :

Un substantif modifié par un adjectif,

Un adjectif modifié par un adverbe,

Un verbe modifié par la proposition qui le régit.

Une telle structuration du texte lisible, qui correspond à ce que dans la conception psychanalytique d'un rêve nous nommons le texte manifeste, perturbe les catégories grammaticales, et efface la fonction prédicative au profit de ce que Kristeva nomme «une assertion infinie » et qui n'est autre, nous y revenons encore, que la conception proprement peircéenne du signe en tant qu'à cause du phénomène d'interprétance qui le constitue il ne cesse de se déplacer sur une

19. Stéphane Mallarmé, \&es mots anglais », in : O.C..., p. 901. 
ligne sans butée autre que le vain pragmatisme de la vie quotidienne qui ne recoupe jamais le texte poétique en fuite perpétuelle.

... à la traduction du «Coup de dés»

Traduire Un coup de dés ne saurait donc se fonder sur la reconstitution d'une quelconque fonction prédicative du texte ni sur le sens linéaire et syntaxique ici bien trop perturbé pour pouvoir nous servir de guide. Aucune traduction qui se vouerait à la reconstitution à tout prix du sens de la linéarité syntaxique de ce texte, ne saurait satisfaire aux exigences de lecture et de traduction qu'il nous impose.

Il faudrait ici se laisser guider par les métaphores du voyage que nous impose l'étymologie du mot traduction. Une langue étrangère, une terre étrangère. Si le texte est bien, comme l'a vu Paul Valéry, une constellation, - prenons ce mot au pied de la lettre nous en avons le droit - projettons-le sur le bleu du ciel. Il y tracera ses figures et que ce soit de la terre de France ou de l'lle anglaise, les yeux levés au ciel le lecteur le lira, le traduira et l'écrira avec les mots de sa terre et de son corps et sous l'angle particulier qu'il doit à sa position historique et géographique ainsi qu'à ses prédispositions pulsionnelles, comme a pu le faire Julia Kristeva.

Elle peut ainsi reformuler la phrase capitale : « un coup de dés jamais n'abolira le hasard» en suivant la voie du signifié (le plan du contenu), en : «une réussite ne peut clore l'inattendu», ou encore «l'ordre ne détruit pas la mouvance, etc... ${ }^{20}$

Telle est bien la première des trois formes de la traduction telles que nous les propose Roman Jakobson ${ }^{21}$ : 1) Rewording, reformulation ou traduction interlinguale. Les deux autres étant respectivement la traduction interlinguale (ou traduction proprement dite) et la traduction intersémiotique (comme par exemple l'illustration d'un texte).

On pourrait toutefois proposer une autre forme de reformulation tout aussi linguistiquement valable, qui ne privilégierait pas le signifié, mais conserverait quelque chose du signifiant quant à la substance de l'expression :

Un coup, deux déjà, mais n'abot lira le hasard

ou bien :

Un coude des jamais nabote liera le hasard ou bien encore :

Un coude déjà met n'abolit râle à czar

« Jeu »! dira-t-on mais l'écriture n'est-elle pas avant tout autre chose le jeu des signifiants et n'est-ce pas dans la dit-mension même du jeu qu'explose le désir et qu'il sollicite une jouissance nous introduisant ainsi au Plaisir du texte ?

Après tout, le petit livre publié sous le titre énigmatique Mots d'heures, Gousses, Rames. The d'Antin Manuscript (edited and annotated by Luis d'Antin

20. Kristeva, p. 270.

21. Roman Jakobson, Essais de linguistique générale, Editions de Minuit, Paris, 1963.

Aspects linguistiques de la traduction p. 78 et ss, 
Van Rooten, Angus and Robertson), à Londres en 1968, s'il n'est pas le modèle à remettre à nos étudiants pour la traduction d'Alice au pays des merveilles ou de la Traversée du miroir, n'est-il pas tout de même beaucoup plus proche de l'esprit carollien que les traductions de Parisot ou même d'Antonin Artaud. Qu'on en juge : qui se douterait que ce qui suit :

Un petit d'un petit

S'étonne aux Halles

Un petit d'un petit

Ah! degrés te fallent

Indolent qui ne sort cesse

Indolent qui ne se mène

Qu'importe un petit d'un petit

Tout Gai de Reguennes.

est en quelque sorte la «traduction 》 de cette charmante comptine :

Humpty Dumpty

Say on a wall.

Humpty Dumpty had a great fall.

And all the king's horses

And all the king's men

Couldn't put Humpty Dumpty

Together again.

$\mathrm{Ne}$ cherchons pas pour le plaisir morne de je ne sais quel dieu normatif quelle serait "la meilleure manière » de traduire, ni à en faire une soi-disant théorie qui ne serait en fin de compte qu'une forme plus ou moins sophistiquée d'une idéologie pratique, essayons plutôt de repérer ce que peut bien être cette opération traduisante en la suivant sur des axes plus logiques qu'idéologiques, à travers tous ses fonctionnements possibles, afin de déterminer quelle peut être sa place dans la formation et le fonctionnement du langage, compte tenu de ceci que le langage à la différence de la parole n'a absolument pas pour fonction de communiquer quoi que ce fût à qui que ce soit.

Une troisième forme de reformulation nous est proposée par Julia Kristeva dans sa lecture de la constellation mallarméenne, non plus cette fois au niveau du signifié de la phrase capitale, mais au niveau de ces unités minimales qu'elle nomme les différentielles signifiantes. Ce que Stéphane Mallarmé nomme dans une perspective tout à fait a-linguistique : les mots. Suivons-la : Un : désigne une totalité indivisible, d'ailleurs effacée vite par ce « deux 》 (en fait le «de » qui vient après 《coup $»)$, « un coup de », et sert de transition vers la pluralité 《un coup de dés $\rightarrow$ un... deux dés ${ }^{22} 》$. Soulignons ici la reformulation de type 2 , à propos du deux (de) dés, lu ainsi par opposition à «un » et qui se mêle à la reformulation plus développée qu'est la reformulation du type suivant.

La reformulation qui s'effectue dans ce court passage se fait par extension dú champ sémantique (du plan du contenu) extension non pas infinie, mais qui englobe d'une part toutes les valeurs qui peuvent être reconnues à telle unité, par exemple le mot « coup », en fonction de ses différents usages dans le texte mallarméen. «Mallarmé emploie souvent ce mot pour désigner la lumière ${ }^{23}$ 》 et

22. Kristeva, Essais de sémiotique littéraire, op. cit., p. 229.

23. Julia Kristeva, Essais de sémiotique littéraire, op. cit., p. 230. 
Kristeva nous indique deux contextes dans lesquels le mot «coup » se rapporte à la lumière : 1) dans "La lumière et les lettres 》: «Tout à coup l'éruptif multiple sursautement de la clarté comme les proches irradiations d'un lever du jour », et 2) dans «Le pitre châtié »: «Hilare or de cymbale à des points irisés. Tout à coup le soleil frappe la nudité ». Mais le texte n'a pas à proprement parler de limites, il est pris dans l'intertexte, de par le phénomène de la valeur (qui intriguait tant Ferdinand de Saussure), et ses fils se prolongent indéfiniment dans le tissu textuel qui voile le monde et la chose, y traçant des figures où l'on peut suivre le destin sémantique des différentielles signifiantes au-delà, bien au-delà, du texte marqué du style mallarméen. Pourquoi donc en effet ne pas suivre, après coup, le «coup », les cheminements de la lumière jusque dans les Védas où celle-ci, tout comme le don poétique, se nomme : usas, aurore.

Ainsi le «coup», de même que «tous les mots» qui « ont plus ou moins, peut-être, un lien de parenté entre eux...» et « gravitent autour de quelque chose de commun » peut-il, non seulement s'apparenter à lumière, mais aussi à «musique et à lumière » et «à heure»- 《heure» que l'on peut rapprocher de l' « heurt» (Igitur). Que le lexicologue ne frémisse pas d'horreur, car si les mots ne fonctionnent pas ainsi dans le dictionnaire, c'est tout de même de cette façon qu'ils fonctionnent dans l'inconscient et pour ce qu'il en est du texte poétique c'est du côté d'une logique de l'inconscient, en tant que cet inconscient est structuré comme un langage qu'il convient d'aller chercher quelques lumières pour nous éclairer dans notre lectécriture poétique.

«Tous les mots ont plus ou moins, peut-être, un lien de parenté qui les unit ${ }^{24}$ ", certes, et par-delà les frontières, peut-être n'y a-t-il pas de mot qui n'entretienne avec quelque autre mot que ce soit une relation quelconque. Les constellations aussi entretiennent entre elles de vagues liens de parenté ne serait-ce que parce qu'on les repère les unes par rapport aux autres et que toutes gravitent autour de quelque chose de commun qui est le point d'où on les observe ? Lorsque leur aspect change ce n'est pas parce que ce ne sont plus les mêmes, mais simplement parce que nous avons changé de point d'observation.

Prenons «Un coup de dés » et traversons la Manche ou l'océan Atlantique et notre complexe signifiant «Un coup de dés» devient alors a throw (ou $a$ cast) of the dice. Que choisir?

Examinons les liens de «coup» avec «throw». Certes \& coup », comme nous l'avons vu ci-dessus, peut être entendu comme le résultat d'une série de transformations à partir d'une structure sous-jacente aisément reconstituable :

« $\mathrm{X}$ lance les dés ». Après trois transformations on parvient à « un coup de dés ». Encore qu'en ce point on puisse se demander l'intérêt de telles transformations à rebours qui ne sont en aucune manière l'inverse des opérations constitutives du texte, si ce n'est peut-être à tenir absolument à la norme que ces opérations de transformation dégagent. Une norme par rapport à laquelle tout message, toute écriture serait plus ou moins mais nécessairement dérivée, même si, comrne c'est le cas pour Mallarmé, la déviance est telle que la structure normative

24. Stéphane Mallarmé, «Les mots anglais \$, in : O.C., p. 918. 
n'est plus reconstituable. Pour nous, la linéarité syntaxique n'est pas première, elle est bien plutôt le résultat de toute une série de contraintes qui ont écrasé et canalisé sur la ligne de la page et l'axe de la monosémie et de la consécution phonétique toutes les sémiotiques originelles beaucoup plus proches du langage mallarméen ou de la langue mystérieuse d'Artaud que des phrases germinatives auxquelles les exercices de la grammaire transformationnelle aboutissent. Nous pensons que les travaux de Leroi-Gourhan sur ce sujet sont particulièrement éclairants en ce qu'il montre bien que le langage ne dérive pas de l'articulation du cri et de la linéarité originelle de la parole, mais qu'au contraire, dès le début, dès les premières traces que l'on peut en retrouver il apparaît déjà comme pluri-sémiotique, comme multiple et comme spatial beaucoup plus que linéaire. Ce n'est d'ailleurs qu'à l'avènement des sociétés agricoles que la linéarité s'est imposée, pour la comptabilité des troupeaux et les débuts de l'accumulation du capital.

Au cours de ces trois transformations de la phrase capitale du poème, le verbe est élidé par suite d'une nomination, mais il n'est pas pour autant supprimé puisqu'on le retrouve plus loin «quand bien même lancé dans des circonstances éternelles $»$, il est déplacé dans la première séquence introduite en incise dans la phrase capitale. Utiliser «throw» ou «cast» (même si ce dernier verbe contient le phonème $/ \mathrm{K} /$ qui se lie au phonème $/ \mathrm{d} / \mathrm{de}$ « dice », permettant ainsi la conservation de l'investissement agressif) aboutirait précisément à ce que parvient à éviter Stéphane Mallarmé : une répétition, qui placerait de surcroît le complexe signifiant à un niveau plus élémentaire dans la série de transformations qui part de la structure sous-jacente. Une telle traduction atténuerait le travail de dé-chaînement, nous dirions plutôt de dé-senchaînement syntaxique auquel se livre Mallarmé. C'est la raison pour laquelle il importe de choisir roll.

Avec roll on retrouve un phonème sombre $/ 0 /$ en opposition au phonème plus clair /ai/ de dice. Opposition qui reprend celle de «coup» et de « dés»: /u/ vs /e/.

Mais examinons roll un peu plus attentivement. A roll of the dice. Le jet des dés, une fois, une révolution des petits cubes marqués s'oppose à never, « jamais». Mais ici par une contrainte inéluctable de la syntaxe anglaise - que Mallarmé ne regretterait certainement pas, «la seule garantie » - les termes de l'opposition sont intervertis : Never will a roll of the dice.

Nous retrouvons le lien qui unit "a roll of the dice» au hazard intacte tant métonymiquement que métaphoriquement. Le Oxford English Dictionary cite l'origine arabe du mot hazard, origine qui peut être le nom d'une ville ou d'un jeu de dés. Ici, au contraire de ce qui se passe dans le texte français, la boucle sémique de la phrase est soulignée par la traduction : never will a roll of the dice abolish hazard, par le sens de roll : to move as in a cycle, évoluer en un cycle : «un coup de dés n'abolira jamais les dés ${ }^{25}$ 》.

Mais on découvre quelque chose d'encore plus remarquable, par le biais de l'association «coup » - «lumière » et de la valeur du mot mythiquement déve-

25. Kristeva, p. 278. 
loppé par son passage à travers le texte de Védas usas : "lumière », "don poétique », le détour fait de ce « coup » l'amorce de l'acte poétique. Or, précisément, et sans faire de détour par les Védas, roll signifie, en tant que substantif : a sonorous or rhythmical flow of words, un flux de mots rythmé ou sonore, n'est-ce pas le poème ? Peut-être ce texte-constellation est-il un accès à la pensée, mais aussi dès la phrase capitale il est accès à l'élan du flux poétique, accès étant à prendre dans son double sens d'accéder, mais aussi de brusque apparition (accès de fièvre), au poème ou mieux encore à la jaculation signifiante : a rhythmical flow of words.

Nous perdons peut-être avec roll les rapports à la lumière, la lumière comme coup, mais nous conservons le rapport au temps «le coup, c'est l'heure » dans Igitur, « le coup s'accomplit, douze, le temps». Mais roll n'est-ce pas aussi to move as time? n'est-ce pas de surcroît la révolution périodique du douze, de l'heure ou des douze heures, la révolution des aiguilles sur le cadran, ou bien encore la révolution des corps célestes dans le ciel sur une orbite?

Roll nous entraîne vers la constellation en mouvement, ce que ce poème « est », très précisément, aux yeux de Paul Valéry. Enfin, et il s'agit là d'une dimension nouvelle que n'offrait pas «coup», roll c'est aussi le roulement de tonnerre et de la tempête « du fond d'un naufrage», le roulement des vagues. Sème qui traverse de part en part tout le poème "Abîme blanchi étale furieux » et sur cette mer déchaînée la coque qui roule bord sur bord et se balance... "béante profondeur en tant que la coque d'un bâtiment penché de l'un ou l'autre bord ». Ainsi roll, dès le début, dès la phrase capitale en anglais, amorce-t-il tout ce thème de la mer et du roulement des vagues qui se poursuit à travers tout le poème pour réapparaître de nœud en nœud : « au nom des flots... naufrage cela... sans nef n'importe où vaine... son ombre puérile caressée et polie et rendue et lavée assoulie par la vague... dans quelque proche tourbillon d'hilarité et d'horreur voltige autour du gouffre... » etc.

Dans le même temps roll impose la jaculation poétique : a sonorous and rhythmical flow of words, comme constellation performing a periodical revolution, as heavenly body. Le poème pris entre l'infini du ciel et de la mer, dans l'infini de la mouvance d'une assertion infinie.

Notons par ailleurs que roll renforce également le sème de vide, de creux, de gouffre que contenait la différentielle signifiante «abol » dans « abolir» et que l'on retrouve dans l'anglais abolish ainsi que le sème de désastre, de naufrage amorcé par le mot « dés», mais que l'on pourrait croire perdu avec dice.

Plus liquide que «coup», roll nous fait perdre «le coup de cymbale du soleil », et la violence agressive et négative, le rejet dont la séquence est investie par les bases pulsionnelles de phonèmes $/ \mathrm{k} /, / \mathrm{d} /, / \mathrm{d} /$ de la suite « coup $d e$ dés ». Dans la traduction la nécessité syntaxique qui nous fait placer never au début de la phrase capitale souligne le rejet sémantiquement. Quant au mot roll disons encore que ses connotations font dériver le poème vers le génie de la langue anglaise en ce qu'il connote l'océanité, tout aussi bien mallarméenne quoique seconde en regard de l'éclat des cymbales et du soleil qu'introduisait le « coup » de la langue française. 
Ces glissements, ces déplacements de connotations ou d'intensités ne constituent pas l'introduction de nouvelles connotations dans la constellation du texte originel, elles ne font que respecter ces constellations autant que les distorsions géographiques le permettent, c'est-à-dire de la plus vaste polysémie possible. Telle facette de l'idée : «lutte de l'ancestral avec la mer » luira peut-être davantage dans la perspective de la langue anglaise que dans celle de la langue française, mais on ne peut finalement que s'en réjouir car le texte global (français et anglais) du coup de dés brillera de mille éclats nouveaux parce qu'auparavant éclipsés par ces éclats propres à la seule langue française.

Il n'est peut-être pas inutile de noter ici que si dans une traduction anglaise, le liquidien l'emporte sur le lumineux, par les nécessités de la syntaxe anglaise, le sème d'insistance qui se trouvait en français dans l'amorce de la première séquence «quand bien même lancé dans des circonstances éternelles du fond d'un naufrage $\gg$ va se trouver déplacé, si tant est qu'on veuille le conserver, vers le complexe signifiant du naufrage : even though cast under eternal conditions from the very core of a shipwreck.

Remarquons que l'agressivité violente, faite de négativité et de rejet, dont nous avions perdu la trace phonique en élidant les phonèmes durs du «coup de dés » dans a roll of the dice se retrouve grâce à la retrouvaille de ces mêmes phonèmes dans : cast under eternal conditions dans la première séquence qui peut être reliée à never : never..., even though cast under...; le sème de rejet restant ainsi lié à celui d'agressivité.

D'où le coup de dés est jeté, par exemple du fond d'un naufrage, il n'y a rien, pas de sujet. Or, ce qui dans les textes français sera formulé plus loin quant à la division du sujet qui est peut-être élidée au début mais que l'on trouve très vite dans « le maître, le vieillard, l'homme, mais à l'ombre puérile 》 apparaît très vite dans le texte anglais. Le sujet, dans ce texte, apparait comme lieu d'un conflit au sein du gouffre marin, conflit qui le fait sans cesse pencher vers la folie, folie qu'il ne fuit pas mais outre-passe par l'écriture, en perdant son statut de sujet, même divisé, pour n'être plus que scripteur, un scripteur qui ne retrouvera nul statut de sujet dans un texte qui le signifierait, car le texte ne s'appuie pas sur le sens, mais sur le "Nombre ». Nombre que menace le délire, la dissolution dans le hasard, la dissociation. Cela même dans notre traduction apparaît dès the very core, le «fond du naufrage », le lieu absolument central, évidé, où se tenait le sujet. Une belle acception moderne du mot est à retenir car elle lui donne dans ce texte toute sa valeur : c'est le cœur creux d'un réacteur où l'on place la matière fissile. D'où les dés sont jetés, par un scripteur, du fond du gouffre où le sujet fait naufrage, là, il n'y a rien que ce jet d'un flux verbal rythmé et sonore au sein d'un processus toujours effectif de dissociation, de désintégration.

Ce tourbillonnement des signes avec la mort pour centre et pour entour, cette jaculation signifiante « jeu» avec les mots «calcul dans le signifiant»par un sujet qui n'est plus homme «mais tout de même sujet-agent 》 (celui qui jette les dés) «levier de la pensée, subjectité » ne reposent sur aucun lieu central : «le texte est la dissolution du lieu central». 
Au fond, tout ceci, a roll of the dice nous le dit déjà. Nous avons vu les sens de roll : flux de mots rythmé et sonore... constellations en orbite autour d'un centre... roulement des vagues, tourbillons, gouffre... et enfin parchemin roulé, texte. $A$ roll of the dice : le Nombre, le calcul des signifiants, la combinatoire plus numérique que significative. Pas de «sens » dans tout cela, pas de signifié... mais de la signifiance. Enfin dice; les dés, mais aussi le pluriel de die. Die, c'est le dé, c'est aussi mourir, n'être plus, c'est le lieu de la dissolution. Le texte comme calcul, organisation des signifiants, constellation certes! mais aussi de dissociation du centre et mort de l'UN.

François Peraldi

Remercions après cette amorce de traduction, Daniel Slote pour son aide et sa compréhension à l'endroit de ce que nous tentions de montrer. 